\title{
Large-Scale Correlations in the Orientation of Grains in Lead-Free Solder Joints
}

\author{
JULIEN SYLVESTRE ${ }^{1,2}$ and ALEXANDRE BLANDER ${ }^{1}$ \\ 1.-IBM Systems \& Technology Group - Global Engineering Solutions, Bromont, QC, Canada. \\ 2.-e-mail: jusylves@ca.ibm.com
}

\begin{abstract}
A large number of lead-free $\mathrm{Sn}-\mathrm{Ag}-\mathrm{Cu}$ controlled collapse chip connection (C4) solder joints ( $\sim 100 \mu \mathrm{m}$ in diameter) in flip-chip microelectronic packages were studied by electron backscatter diffraction (EBSD) in order to describe the statistical distribution of grain size and coincident site lattice boundaries associated with $60 \mathrm{deg}$ twins in the Sn phase, as a function of silver content. It is shown that lower silver content results in smaller grains, and a lower propensity for grains to exhibit twinning symmetries. Indirect measurements of the creep properties of these joints were also obtained as a function of silver content, showing that, in the strain rate and temperature conditions that are the most relevant to the microelectronic industry, solder joints with low silver content are more susceptible to creep deformation.
\end{abstract}

Key words: Sn-Ag-Cu, solder joints, lead-free, microstructure, plastic creep, grain size, twinned grain boundaries

\section{INTRODUCTION}

The recent adoption of more stringent legislation to protect the environment from lead contamination is driving a worldwide microelectronic industry effort to introduce lead-free solder systems. In spite of the large amount of literature published on these alloys, their use in small structures such as controlled collapse chip connection (C4) solder joints in flip-chip packages results in a largely unexplained variability in their mechanical and diffusive properties. ${ }^{1}$ The small number of grains in lead-free solder joints ${ }^{2}$ and the dependence of the microstructure on the thermal or process history, ${ }^{3}$ physical size, ${ }^{4}$ and chemical composition ${ }^{5}$ are typically invoked to explain this variability, in light of the fact that tin is highly anisotropic in its elastic response $^{6}$ and creep properties. ${ }^{7}$

The creep properties of solder joints are especially important during the reflow used to join the semiconductor device to substrates, where large stresses can develop in the joints due to the difference in thermal expansion between device and substrate,

(Received December 14, 2007; accepted June 6, 2008;

published online July 2, 2008) leading to mechanical damage in the device. ${ }^{8-10}$ Finite element models based on creep are also frequently invoked to explain fatigue data in thermal cycling during reliability testing. ${ }^{11}$ It is generally assumed, however, that a complete understanding of the mechanical characteristics of solder joints will require a proper description of the anisotropic properties of the small number of grains forming the joints. ${ }^{12}$ Such an understanding will be especially useful if it can be combined with a statistical description of the microstructure in lead-free joints, which should include the number of grains, their size and orientation, as well as the correlations in the orientation of these grains. Such a statistical description may allow for the optimization of the solder alloy and structure in a way that encourages robustness in a manufacturing environment, where enormous numbers of solder joints are produced.

The question of the statistical description of the microstructure of solder joints has been partially addressed in the literature. It has been shown for instance that solder intermetallic compounds grow with a preferred orientation relative to the underlying solder pad. ${ }^{13}$ Closer to the point of this paper, the presence of twins in the $\beta$-tin phase in $\mathrm{Sn}-\mathrm{Ag}-\mathrm{Cu}$ solder joints has been frequently reported. Beach 
ball twinning is typically comprised of (101))[101] twins having a $58 \mathrm{deg}$ to $59 \mathrm{deg}$ tilt boundary. When a single twinning axis is present, a cross-section of the joint can reveal six sectors (pie section shaped) each with an orientation differing by approximately $60 \mathrm{deg}$ from its neighbors, producing a characteristic image, when orientation is color coded, which resembles a beach ball. ${ }^{14}$ Interlaced twins have been reported as fine-grained (301)[103] twins having tilt grain boundaries of $\sim 62$ deg. ${ }^{15}$

For both types of twins, the two crystals spanning the twin boundary are rotated by the twinning angle ( $\sim 58 \mathrm{deg}$ or $\sim 62 \mathrm{deg}$ ) about a common [100] crystal direction. As such the (101) or (301) planes are shared between adjacent crystals and are thus termed the twin plane. Both types of twins have the characteristics of perfect atomic sharing at the twin plane, resulting in a coherent boundary. It must be noted, however, that if in the case of (101)[101] twins, six grains were each to share the same twin axis, the twin boundary would not be perfectly coherent or strain free, as misfit would have to accommodate the gap imposed by six $\sim 58 \mathrm{deg}$ boundaries. Both the (101)[101] and the (301)[103] twinning systems can be explained by a stacking fault occurring in the stacking of the sequence of (101) or (301) planes, respectively. It can be shown that an ABCDABCDCBADCBA type of stacking fault is responsible for both twinning systems, with the underlined $\mathrm{D}$ atomic plane being the twin plane, common to both lattices at the boundary. The two twinning systems will be treated together for the reporting of the analysis results below, in the sense that the designation $60 \mathrm{deg}$ twin will represent indiscriminately either of the two coherent twin boundaries described above.

We have studied a large number of small ( $\sim 100 \mu \mathrm{m}$ in diameter) lead-free solder joints using electron backscatter diffraction (EBSD) imaging in order to measure the distribution of grain size and the degree of twin boundaries as a function of various parameters. The analysis of these data was highly automated, hence a large dataset consisting of a large number of grain pairs $(N=1252)$ could be assembled. We report here on the influence of chemical composition on the texture of $\mathrm{Sn}-\mathrm{Ag}-\mathrm{Cu}$ solder joints. As the amount of Ag in the solder is reduced, the number of grains in the solder joint increases. In addition, the reduction of $\mathrm{Ag}$ content leads to a reduction in the number of $60 \mathrm{deg}$ twins observed in the joints. Solder joints built with a $0 \% \mathrm{Ag}$ metallurgy have a very small number of twins, while metallurgies with Ag content by weight above $2 \%$ have more than $70 \%$ of grains which are twinned.

In parallel to these texture studies, we measured creep in real solder joints, again for $\mathrm{Sn}-\mathrm{Ag}-\mathrm{Cu}$ metallurgies with varying $\mathrm{Ag}$ content. Creep is measured indirectly in actual microelectronic packages, under realistic reflow conditions for the initiation of damage to the device (temperature: $\sim 25^{\circ} \mathrm{C}$, creep rate: $\sim 10^{-7} \mathrm{~s}^{-1}$ ). These data clearly show a progressive increase in the propensity to creep with the progressive elimination of $\mathrm{Ag}$. In a model where grain boundary sliding plays a significant role in the creep deformation of these solder joints, these EBSD and creep measurement data would be compatible with the suppression of creep deformation by the presence of coherent twin boundaries between grains.

\section{EBSD OBSERVATIONS}

Flip-chip plastic ball grid array (FCPBGA) devices were mounted, sectioned, ground, and polished to reveal the $\mathrm{Sn}-\mathrm{Ag}-\mathrm{Cu}$ solder joints. To prepare the surface for EBSD, samples underwent ion milling in a Fischione 1030 automated sample preparation (ASaP) system. EBSD analysis was performed on a Hitachi 4700 cold field-emission gun (FEG) scanning electron microscope (SEM), coupled with an Oxford Instruments EBSD camera with INCA Crystal software. The pixel area of most EBSD scans was $2.76 \mu \mathrm{m}^{2}$, which led to a pixel step size of approximately $1.66 \mu \mathrm{m}$. Standard noise reduction techniques, such as nearest-neighbor pixel dilation and thresholding of a minimum pattern quality, were employed to remove artificial pixel effects caused by non- $\beta$-Sn phases present in the microstructure.

Misorientation maps between neighboring pixels were generated in postprocessing, in order to quantify the difference in orientation between a pixel and its four natural neighbors. More precisely, given two pixels with associated orientation data each parameterized by a distinct set of three Euler angles defining an orientation matrix, a third set of Euler angles was calculated to describe the rotation matrix which would bring both adjacent lattices into coincidence, by rotating the orientation matrix of the first pixel into the orientation matrix of the second pixel. We used the Cardan convention for the Euler angles, so that two angles described a direction in space (the rotational axis) around which the rotation by the third angle occurred (thus providing an angle/axis pair parameterization). This third angle is simply called the misorientation angle between two pixels. The misorientation angle was used to form the misorientation map, with every pixel in this map being the maximum of the misorientation angle over the four natural neighbors of the pixel. Symmetries of the $\beta$-Sn tetragonal unit cell were properly incorporated to maintain values of the misorientation map between 0 deg and $90 \mathrm{deg}$.

The misorientation map for every solder joint was partitioned into simply connected domains with maximum misorientation between neighboring pixels smaller than $15 \mathrm{deg}$. Due to the presence of noise in the images and to the relatively coarse spatial resolution of the EBSD maps, some grains were broken up into more than one cluster, such that a reconstruction analysis was required to correctly 

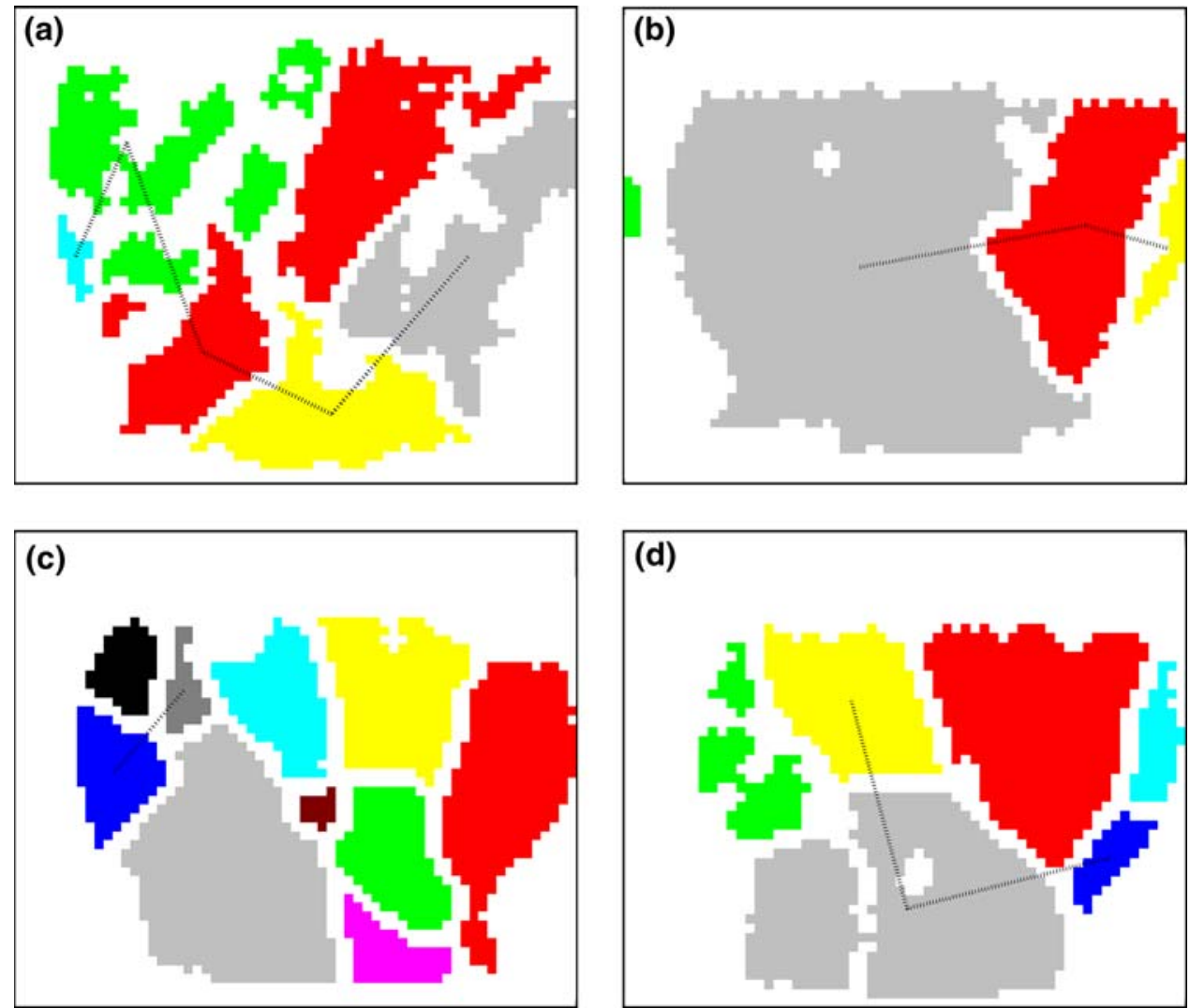

Fig. 1. Color maps: each color represents a unique grain domain; 60 deg twin boundaries are identified by dotted lines which connect the center of masses of regions associated with a unique orientation. $(\mathrm{a}, \mathrm{b})$ Examples of SAC solder system with $2.2 \%$ Ag, note in (a) the "reconstruction" feature of the classification used in the SAC system, where many disjoint clusters are joined into single grains (single colors). (c, d) Two examples of a $0 \% \mathrm{Ag}$ system. Note the sparsity of $60 \mathrm{deg}$ twins in $0 \% \mathrm{Ag}$ solder as compared with the $2.2 \% \mathrm{Ag}$ solder.

identify unique grains. Starting from a set of pixel clusters in an EBSD image, a connectivity graph was constructed to define the spatial relationship between the clusters. The graph is the set of all cluster pairs in the EBSD map that are natural neighbors. The concept of natural neighbors is somewhat subjective as it is related to clusters in a noisy image which could potentially be parts of a single grain.

Once the connectivity graph is constructed, each cluster pair is considered in turn in order to classify the nature of the relationship between the members of the pair. A large number $(\sim 100,000)$ of independent pairs of pixels are generated by randomly selecting one pixel at a time in each of the two clusters being examined. For each pixel pair, the misorientation angle is calculated (from the initial EBSD map) as described above, and the histograms of all misorientation angles and directions for that pair of clusters are constructed. These histograms are then used to classify the pair of clusters.

If the misorientation angle averaged over all pixel pairs is smaller than $15 \mathrm{deg}$, the clusters are considered to be part of a single grain which has been broken up by noise. Various coincident site lattice (CSL) boundaries can also be considered by the system. In all cases, the classification requires an average misorientation angle within \pm 5 deg of the CSL rotation angle, and $95 \%$ of the pixel pairs to have a rotational axis which is within $10 \mathrm{deg}$ of the CSL rotational axis. Only $60 \mathrm{deg}$ rotations about the $a$ or $b$ axis of the Sn unit cell will be reported here, as they are much more numerous in our dataset than other CSL boundaries known to exist in Sn. ${ }^{16}$

Colored maps can be constructed from this classification strategy in order to describe efficiently the microstructure of a solder joint with respect to grain size and CSL boundaries. Colors are used to uniquely define distinct grains, in the sense that clusters of the same color are part of the same grain and share a common orientation which is clearly distinguishable from the orientation of neighboring grains. Additionally, dotted lines between the center of mass of clusters identify 60 deg twins. See Fig. 1 for examples.

\section{TEXTURE DATA}

A total of $134 \mathrm{C} 4$ solder joints (approximately $100 \mu \mathrm{m}$ in diameter) were imaged by EBSD. In all cases, the joints were formed by assembling a semiconductor device on an organic laminate substrate. Before joining the device to the substrate, a 
Table I. Summary of Observations (See Text for Details)

\begin{tabular}{|c|c|c|c|c|c|}
\hline $\begin{array}{l}\text { Ag Content } \\
(\text { wt. } \%)\end{array}$ & $\begin{array}{l}\text { Number } \\
\text { of Solder } \\
\text { Joints }\end{array}$ & $\begin{array}{c}\text { Number } \\
\text { of Distinct } \\
\text { Grains }\end{array}$ & $\begin{array}{l}\text { Number } \\
\text { of Grains } \\
\text { Per Joint }\end{array}$ & $\begin{array}{c}\text { Number } \\
\text { of Independent } \\
\text { Pairs }\end{array}$ & $\begin{array}{c}\text { Fraction } \\
\text { of Pairs Being } \\
60^{\circ} \text { Twins }(\%)\end{array}$ \\
\hline 2.2 & 54 & 241 & $4.5 \pm 0.56$ & 250 & $\begin{array}{l}71_{-6.0}^{+5.5} \\
(178)\end{array}$ \\
\hline 1.3 & 10 & 52 & $5.2_{-1.3}^{+1.6}$ & 59 & $\begin{array}{c}44_{-13}^{+14} \\
(26)\end{array}$ \\
\hline 0.3 & 10 & 112 & $11.2 \pm 2.1$ & 199 & $\begin{array}{c}8.5_{-3.5}^{+4.8} \\
(17)\end{array}$ \\
\hline 0.0 & 60 & 471 & $7.9 \pm 0.71$ & 744 & $\begin{array}{c}19_{-6.1}^{+7.4} \\
(28)\end{array}$ \\
\hline
\end{tabular}

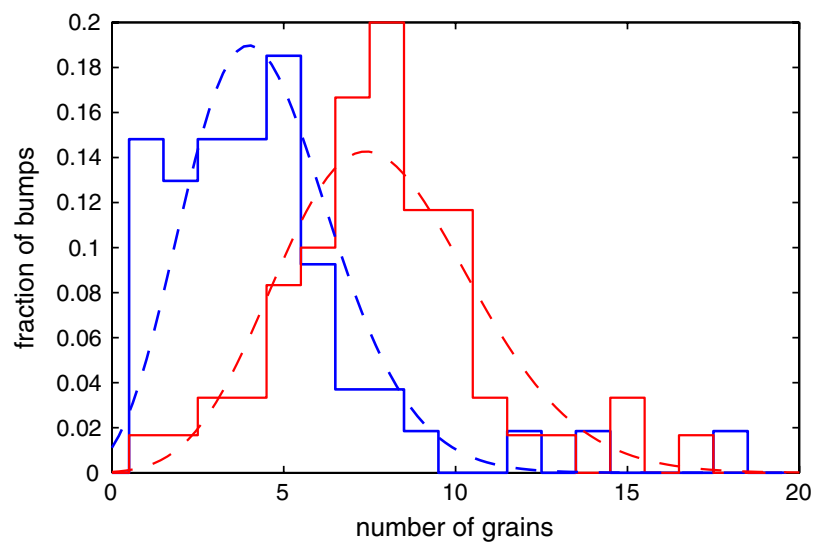

Fig. 2. Histograms for the number of independent grains, with fits to the Poisson distribution (dashed lines). Leftmost curves (blue-color online): $2.2 \% \mathrm{Ag}$. Rightmost curves (red-color online): $0 \% \mathrm{Ag}$.

certain quantity of solder was deposited on the barrier layer metallization (BLM) on the device and on the solder pads on the substrate. The solder alloy on the device was either $\mathrm{SnCu}$ or $\mathrm{SnAg}$, while $\mathrm{SnCu}$ or $\mathrm{SnAgCu}$ was deposited on the substrate. The exact metallurgy of the joint after chip joining is a complex mixture of alloys defined by the interdiffusion of the BLM, solder joints, and the laminate, and in general has to be measured to be determined precisely.

Table I shows the important parameters measured from these observations. The first column is the approximate weight percent of silver in the final joint. The second column is the number of solder joints imaged. The third column is the total number of distinct grains, while the fourth column is the mean number of distinct grains per joint (maximum likelihood of the parameter of a Poisson distribution), together with 95\% confidence intervals. Figure 2 shows how the Poisson distribution fits the data for the number of distinct grains (note that the variance of a Poisson deviate is equal to its mean). The fifth column is the total number of independent grain pairs observed, and the last column gives the mean fraction of independent grain pairs which are $60 \mathrm{deg}$ twin boundaries. The maximum-likelihood fraction (assuming a binomial distribution) is

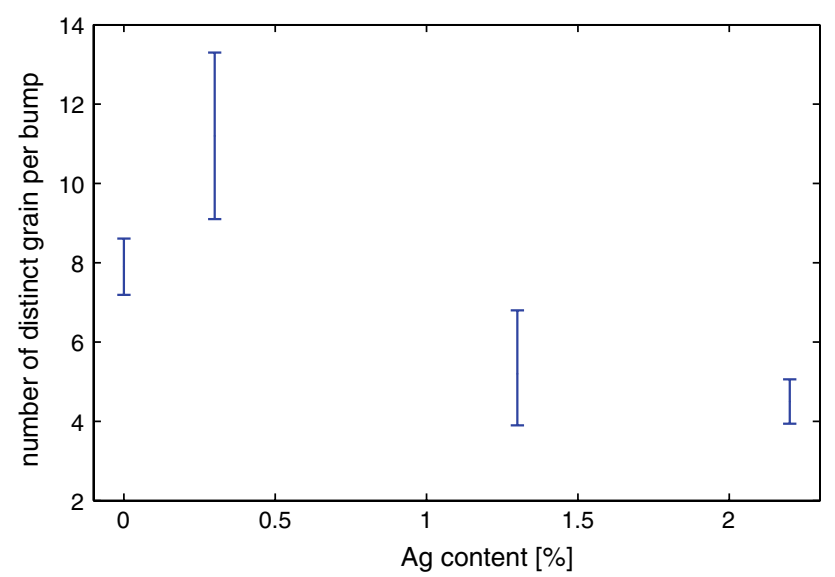

Fig. 3. Poisson parameter with $95 \%$ confidence interval for the number of distinct grain per joint, as a function of silver weight content.

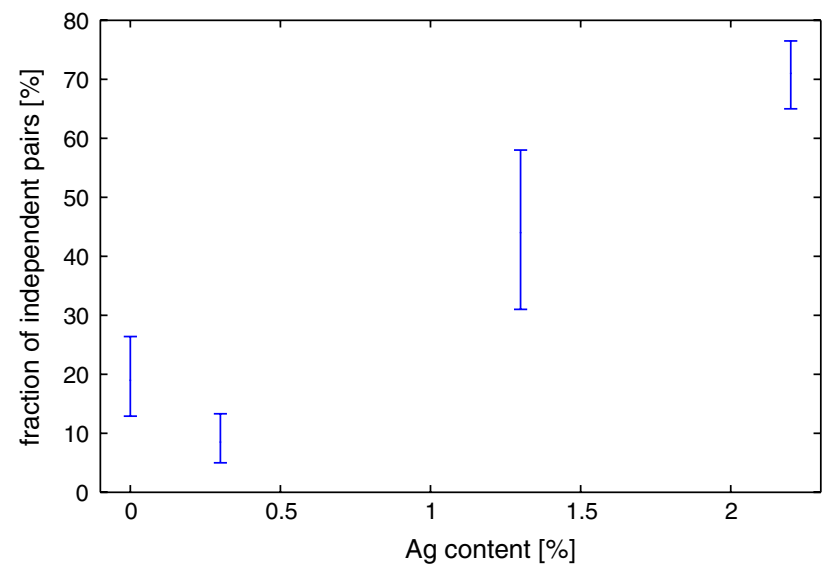

Fig. 4. Fraction of independent grain pairs with a 60 deg coherent twin boundary, with $95 \%$ confidence interval (from the maximumlikelihood estimator of a binomial distribution), as a function of $\mathrm{Ag}$ weight content.

reported, together with $95 \%$ confidence intervals and raw number of observations in parentheses.

Figures 3 and 4 show how the silver concentration in the joint affects both the distribution of grain size and the presence of the coherent $60 \mathrm{deg}$ twin boundaries between grains. The $2.2 \% \mathrm{Ag}$ joints 
typically contained $4.5 \pm 2.1$ grains ( \pm 1 standard deviation), and roughly $71 \%$ of all grain boundaries were $60 \mathrm{deg}$ twin boundaries. At the other extreme, $\mathrm{SnCu}$ joints had significantly more grains $(7.9 \pm 2.8)$, wherein these grains did not have the same tendency to be related by twin symmetries that were observed for higher silver joints. Other CSL boundaries were observed (predominantly around $80 \mathrm{deg}$ ), but their statistics were not sufficient to draw conclusions on the impact of $\mathrm{Ag}$ content on their rate of occurrence.

\section{DYNAMIC CHIP WARPAGE MEASUREMENTS}

The mechanical properties of lead-free solder alloys are known to be very sensitive to the physical size of the measured sample, to chemical composition, and to its thermal history. Because real solder joints have properties which are hard to emulate in a test sample (exact composition after dissolution of material from the solder pads, small size and geometry, reflow conditions, etc.), it is desirable to measure their mechanical properties in situ, on real devices.

A measurement system was developed for the semiquantitative estimation of the creep properties of lead-free solder joints which is both reasonably precise and very cost effective to implement in a real manufacturing environment. The system dynamically measures the deformation of the back side of the semiconductor device in a flip-chip package using a laser interferometer at a high sampling rate (a few $\mathrm{Hz}$ ) and high resolution $(\sim \mu \mathrm{m})$, allowing the synchronization of the chip warpage data and of thermal data during a chip joining reflow cycle. The difference in thermal expansion between the device [coefficient of thermal expansion $(\mathrm{CTE}) \sim 4 \mathrm{ppm} /{ }^{\circ} \mathrm{C}$ ] and the organic substrate $\left(\mathrm{CTE} \sim 20 \mathrm{ppm} /{ }^{\circ} \mathrm{C}\right)$ imposes significant strains on the solder joints, which can be shown to be a function of several process parameters. ${ }^{10}$ The data reported here were collected by varying the Ag content of the solder joints, keeping all other process parameters constant. As argued in Ref. ${ }^{10}$, the maximum chip warpage during the reflow, which is the quantity reported here, is a good approximation to the flow stress from creep deformation. This maximum warpage occurs near room temperature during the cooling portion of the reflow, when the strain rate is approximately $10^{-7} \mathrm{~s}^{-1}$.

Figure 5 shows the maximum chip warpage during a standard reflow cycle, as a function of silver content. It is quite interesting to notice that the propensity to creep varies in the same manner as the number of grains and inversely to the fraction of $60 \mathrm{deg}$ twin boundaries in the joint population when the concentration of silver is reduced. This observation would be supported by a model for creep where grain boundary sliding plays a significant role. It is indeed quite plausible that the increased

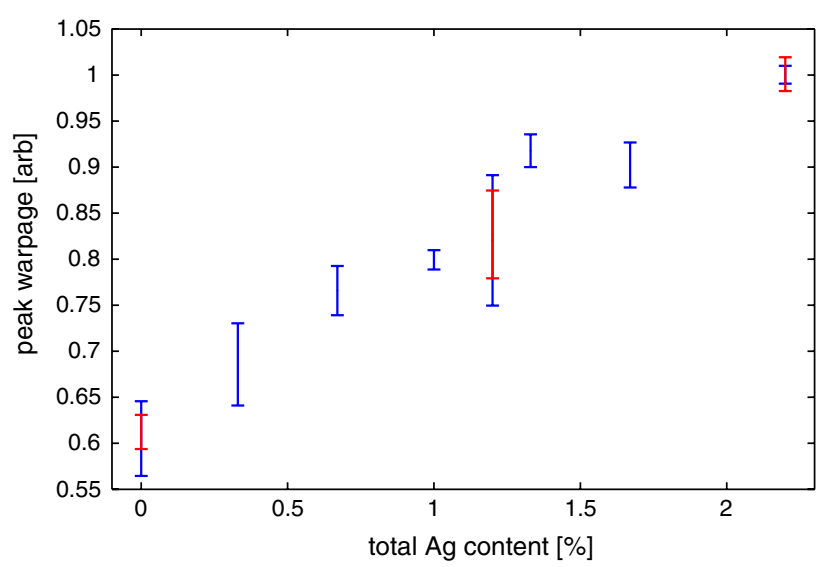

Fig. 5. Maximum chip warpage during a standard reflow (arbitrary units), with $95 \%$ confidence intervals, as a function of $\mathrm{Ag}$ weight content. Overlapping bars (red and blue online) correspond to measurements on two different flip-chip packages with different chip dimensions and substrate thicknesses.

propensity of lower Ag alloys to creep results from the increased grain boundary area through grain refinement, and from the reduced fraction of grain boundaries which are highly coherent, low-energy twin boundaries.

This hypothesis could be verified experimentally in many different ways, for instance by carrying out nondestructive creep tests on very small samples, which could further be characterized by EBSD to correlate their creep response to the total area of grain boundaries and the presence of twin boundaries. Indirect evidence supporting this hypothesis can also be found in the literature. For instance, it has already been observed that smaller grains could be related to higher creep rates (grain refinement alone is unlikely to explain the large observed variation in creep resistance; eliminating Ag from the alloy increased the number of grains by a factor of $\sim 2$ in our measurements, while creep is generally observed to scale weakly with grain size in similar alloys ${ }^{17}$ ). In addition, the stress exponent calculated from fits to creep data has frequently been reported to increase from a low value ( 2) typical of grain boundary sliding at low strain rates, to a higher value ( 10) typical of dislocation climbing at higher strain rate. ${ }^{18}$ Our data are consistent with these observations on $\mathrm{Sn}-\mathrm{Ag}-\mathrm{Cu}$, as well as with observations on other metals that grain boundary sliding is inhibited by the presence of boundaries with coincident site lattice orientations. ${ }^{19}$

On the other hand, intermetallic compounds (IMC), if they play a significant role in the pinning of dislocations in $\mathrm{Sn}-\mathrm{Ag}-\mathrm{Cu}$, could be the basis of a dislocation model to also explain our creep deformation data, either for the case of Ag that is uniformly distributed in small precipitates within the solder joint, or for the case where it is preferentially located at grain boundaries. In both cases, in the absence of significant grain boundary sliding, the dependence of creep resistance, $60 \mathrm{deg}$ twins, and 
grain size on $\mathrm{Ag}$ reported here would be largely coincidental.

\section{CONCLUSIONS}

The microstructure and creep properties of $\mathrm{C} 4 \mathrm{Sn}$ $\mathrm{Ag}-\mathrm{Cu}$ solder joints $(\sim 100 \mu \mathrm{m}$ in diameter) were studied as a function of $\mathrm{Ag}$ content. By analyzing a large number of joints $(N=134)$ using EBSD imaging supported by a highly automated classification analysis, it was shown that lower Ag contents resulted in smaller, more numerous grains, as well as fewer coherent twins in the resulting solder joints. This implies that Ag plays an important role either at the nucleation or growth stages of solidification by impacting whether the microstructure will be highly oriented and large grained. The detailed mechanism by which $\mathrm{Ag}$ influences texture in SAC is not properly understood, and should be further investigated.

In parallel, a semiquantitative study of the creep properties of these solder joints under realistic loading conditions revealed that lower $\mathrm{Ag}$ contents resulted in solder joints that had a lower resistance to creep deformation. These results provide an important example of the influence of the microstructure on the mechanical properties of these solder joints. They are especially interesting as they support the hypothesis that grain boundary sliding is the dominant mechanism for creep deformation near room temperature at low strain rates in $\mathrm{Sn}-\mathrm{Ag}-\mathrm{Cu}$ solder joints, with the sliding being facilitated by the increased grain boundary area and the lower coherence of nontwinned boundaries in lower Ag alloys.

The proper understanding of the influence of the solder joint microstructure on its mechanical properties is of tremendous importance to the microelectronic industry in that it would allow the development of more precise reliability models of these joints under various stress conditions. In addition to the savings in stress testing resources that this would allow, these models would provide invaluable insights into the development of new interconnection technologies, such as finer-pitch interconnects and alternative alloys. In order to fully realize the potential of these models, however, the research community will have to maintain a clear focus on two key aspects of the problem: a proper description of the anisotropic properties of inhomogeneous joints (including the elastic response, creep, thermocycling fatigue, and electromigration resistance), and a complete statistical characterization of grain shapes and grain boundary coherence.

\section{ACKNOWLEDGEMENTS}

The authors are grateful to Donald W. Henderson for sharing his vast expertise on the subject, in particular on models for the influence of $\mathrm{Ag}$ on the creep behavior. The authors also acknowledge the careful review of this manuscript by Brian $R$. Sundlof, David Danovitch, and Peter Brofman. This work was partially accomplished using the facilities at Hydro-Quebec, with the help of Pierre Hovington.

\section{REFERENCES}

1. F. Yang and J.C.M. Li, J. Mater. Sci. Mater. Electron. 18, 191 (2007).

2. D.W. Henderson et al., J. Mater. Res. 19, 1608 (2004). doi:10.1557/JMR.2004.0222.

3. K.S. Kim, S.H. Huh, and K. Suganuma, Mater. Sci. Eng. A 333, 106 (2002). doi:10.1016/S0921-5093(01)01828-7.

4. S. Wiese et al., IEEE Proceedings of the 57th Electronic Components and Technology Conference (ECTC'07) (2007), pp. 548-557.

5. T.-M.K. Korhonen, J. Electron. Mater. 33, 1581 (2004). doi:10.1007/s11664-004-0101-2.

6. J.A. Rayne and B.S. Chandrasekhar, Phys. Rev. 120, 1658 (1960). doi:10.1103/PhysRev.120.1658.

7. A.U. Telang, T.R. Bieler, D.E. Mason, and K.N. Subramanian, J. Electron. Mater. 32, 1455 (2003). doi:10.1007/s11664-0030115-1.

8. C. Odegard et al., IEEE Proceedings of the 55th Electronic Components and Technology Conference (ECTC'05) (2005), pp. 1163-1171.

9. M. Uchida et al., IEEE Proceedings of the 57th Electronic Components and Technology Conference (ECTC'07) (2007), pp. 885-891.

10. J. Sylvestre, A. Blander, V. Oberson, E. Perfecto, and K. Srivastava, IEEE Proceedings of the 58th Electronic Components and Technology Conference (ECTC'08) (2008).

11. R. Darveaux, Trans. ASME J. Electron. Packag. 124, 147 (2002).

12. J.G. Lee, A. Telang, K.N. Subramanian, and T.R. Bieler, J. Electron. Mater 31, 1152 (2002). doi:10.1007/s11664-0020004-z.

13. J.O. Suh et al., IEEE Proceedings of the 56th Electronic Components and Technology Conference (ECTC'06) (2006), 4 pp.

14. T.R. Bieler et al., IEEE Proceedings of the 56th Electronic Components and Technology Conference (ECTC'06) (2006), pp. 1462-1467.

15. L.P. Lehman et al., J. Electron. Mater. 33, 1429 (2004). doi:10.1007/s11664-004-0083-0.

16. A.U. Telang and T.R. Bieler, J. Electron. Mater. 57, 44 (2005).

17. S. Yi, G.X. Luo, and K.S. Chian, J. Electron. Packag. 124, 91 (2002). doi:10.1115/1.1451845.

18. P.P. Jud, G. Grossman, U. Sennhauser, and P.J. Uggowitzer, J. Electron. Mater. 34, 1206 (2005).

19. G. Wirmark, J.-O. Nilsson, and G.L. Dunlop, Philos. Mag. A 1, 93 (1981). doi:10.1080/01418618108239395. 